\title{
Length measurement in accelerated systems
}

\author{
Bahram Mashhoon and Uwe Muench \\ Department of Physics \& Astronomy, University of Missouri-Columbia, Columbia, Missouri \\ 65211, USA \\ MashhoonB@missouri.edu
}

Received dd.mm.yyyy, accepted dd.mm.yyyy by ue

\begin{abstract}
We investigate the limitations of length measurements by accelerated observers in Minkowski spacetime brought about via the hypothesis of locality, namely, the assumption that an accelerated observer at each instant is equivalent to an otherwise identical momentarily comoving inertial observer. We find that consistency can be achieved only in a rather limited neighborhood around the observer with linear dimensions that are negligibly small compared to the characteristic acceleration length of the observer. Files length1.tex, 2002-06-24
\end{abstract}

Keywords: acceleration, length measurement

PACS: $03.30 .+\mathrm{p}, 04.20 . \mathrm{Cv}$

\section{Introduction}

The primary measurements in physics are the determinations of spatial distances and temporal durations that are associated with the effective establishment of a sufficiently local frame of reference. This process involves macrophysical determinations associated with the fact that physical observers and their frames of reference obey the laws of classical (i.e. nonquantum) physics. The basic nongravitational laws of physics refer to ideal inertial observers; their measurements are briefly discussed in section 2 On the other hand, actual observers are all (more or less) noninertial, i.e. accelerated. In fact, most experiments are performed in laboratories fixed on the Earth, which - among other motions - rotates about its axis; therefore, it is necessary to give a theoretical description of the measurements of accelerated observers. This is done via the hypothesis of locality described in section 3. This hypothesis in effect replaces the accelerated observer by a continuous infinity of hypothetical momentarily comoving inertial observers. Sections 1 and f deal with the measurement of length by observers undergoing translational and rotational accelerations, respectively. Section 6 contains a discussion of our results.

\section{Simultaneity and length measurements}

We begin by reviewing some basic concepts and terms about length measurement that are commonly used for inertial systems in special relativity (SR). 
An event in SR is associated with a single location in space and a single instant in time. The position of an event is defined to be the coordinate label on a rigid ruler that extends from the spatial origin to the event; this notion is then naturally extended to the spatial coordinates that characterize the location of the event in space. The ruler is envisioned to extend indefinitely from some chosen origin. Such a choice is only possible in a global inertial coordinate frame, which can be defined only in Minkowski spacetime for inertial observers. The time of an event is most naturally defined as the reading on a clock located at the event's position at the instant at which the event occurs. The rulers and clocks used by an inertial observer are at rest relative to the observer. Time is somehow a difficult notion to grasp, especially when it becomes frame dependent under Lorentz transformations [1].

\section{Simultaneity}

All inertial observers in SR are assumed to be either actual observers or measuring devices that use synchronized clocks. To determine the time of a distant event, an observer corrects for the travel time of a signal originating at the event. To perform this correction the observer has to know the distance to the event by either determining the event's spatial coordinates in its reference frame or by prior measurement of the distance. The determination of the location and the time of an event are independent of the position of an observer compared to all other observers in the same reference frame.

The time ordering of the events depends on the relative velocity of the inertial observers and the relative position of the events, but not the positions of the observers since global synchronization of clocks is assumed. The invariance of the speed of light $c$ has an additional immediate implication: Two events at different locations that occur at the same time in a given inertial frame are not simultaneous in any other inertial frame. Moreover, $v<c$ for any observer implies that the causal sequence of events is independent of the inertial observers.

Length measurements

An inertial frame is globally defined, since the lifetime of clocks can be ideally extended indefinitely and the rulers ideally extend indefinitely in space. Hence, lengths are simply determined by the differences of the coordinate positions of the endpoint of line segments at the same time in such a reference frame, i.e. $L=\left|\vec{x}_{2}-\vec{x}_{1}\right|$ is the length of the straight line segment extending from $\vec{x}_{1}$ to $\vec{x}_{2}$. In effect, the homogeneity and isotropy of spacetime in an inertial frame allows us to sum intervals of time and space corresponding to the use of finite clocks and rulers.

A ruler of length $l_{0}$ at rest in an inertial frame contracts by a factor of

$$
\gamma^{-1}=\sqrt{1-\beta^{2}}
$$

as measured by standard observers at rest in an inertial frame moving with speed $v=\beta c$ along the direction defined by the ruler; this effect is known as the LorentzFitzgerald contraction.

It is possible to define the distance between two inertial observers using electromagnetic signals: One observer at rest at $\vec{x}_{1}$ in some inertial frame sends out a light signal towards a second (possibly moving) observer. The second observer at $\vec{x}_{2}$ sends 
a light signal back immediately after reception of the first light signal. The first observer determines the time difference $\Delta t$ between sending the first light signal out and receiving the second light signal at its position. The length between the observers is then given by

$$
L^{*}:=\frac{1}{2} c \Delta t
$$

This length definition relies only upon the assumption that the speed of light is constant and equal to $c$ in all inertial reference frames; moreover it is consistent with the measurement of length based on rulers (i.e. $L^{*}=L$ ).

Translational and rotational accelerations

An inertial observer is an ideal that cannot be realized in practice. All actual observers are accelerated. To develop the theory of accelerated systems, let us define an orthonormal frame field $e_{\alpha}$ for an accelerated observer. The components of the frame field are $\lambda^{\mu}{ }_{(\alpha)}:=e^{\mu}{ }_{\alpha}$, where $e_{\alpha}=e^{\mu}{ }_{\alpha} \partial_{\mu}$. We choose $e_{0}$ to be the unit vector $u^{\mu}(\tau):=\frac{1}{c} \frac{d x^{\mu}}{d \tau}$ that is tangent to the worldline at a given event $x^{\mu}(\tau)$ and we parametrize the remaining frame vectors characterizing the spatial directions also by $\tau$, which is a temporal parameter measured along the accelerated path by the standard (static inertial) observers in the underlying global inertial frame according to the formula $\tau=\int \sqrt{1-\beta^{2}(t)} d t$.

The condition of orthonormality for the frame field reads

$$
\eta_{\mu \nu} \lambda_{(\alpha)}^{\mu}(\tau) \lambda_{(\beta)}^{\nu}(\tau)=\eta_{\alpha \beta}=\operatorname{diag}(-1,+1,+1,+1) .
$$

The derivative of the frame field along the accelerated path can be expressed in the frame basis:

$$
\frac{d \lambda^{\mu}(\alpha)}{d \tau}=\Phi_{\alpha}{ }^{\beta}(\tau) \lambda^{\mu}{ }_{(\beta)} .
$$

Using the orthonormality condition, we find that $\Phi_{\alpha \beta}$ is antisymmetric

$$
\Phi_{\alpha \beta}(\tau)=-\Phi_{\beta \alpha}(\tau) ;
$$

we therefore define

$$
\Phi_{\alpha \beta}:=\left[\begin{array}{c|c}
0 & \vec{a} / c \\
\hline-\vec{a} / c & \vec{\Omega}
\end{array}\right],
$$

where $\Phi_{0 i}=a_{i} / c$ and $\Phi_{i j}=\epsilon_{i j k} \Omega^{k}$. Here $\vec{a}$ represents the "electric" component and is the translational acceleration, while $\vec{\Omega}$ represents the "magnetic" component and is the rotational frequency of the local spatial frame (with respect to the local nonrotating, i.e. Fermi-Walker transported, axes).

Let us now introduce a geodesic coordinate system $X^{\mu}$ in the neighborhood of the accelerated path. At any time $\tau$ along the accelerated worldline (see figure (1), 


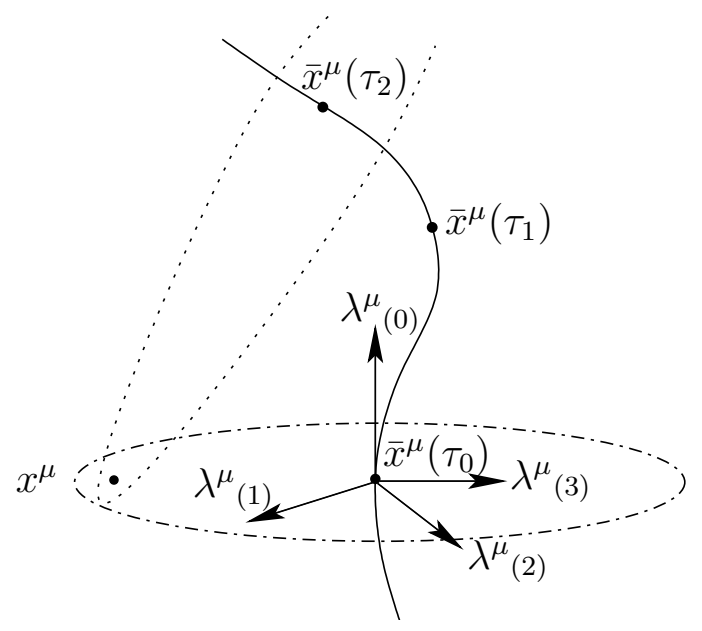

Fig. 1 An event $x^{\mu}$ as seen by the observer $\bar{x}^{\mu}\left(\tau_{0}\right)$ with its frame field $\lambda^{\mu}{ }_{(\alpha)}$. The geodesic coordinate system $X^{\mu}=(c \tau, \vec{X})$ is limited in space: If we go beyond the time $\tau_{1}$, for example, coordinate assignments would start to overlap, as shown for the time $\tau_{2}$. Since this cannot be accepted, spatial coordinates have to be limited in general. Thus the geodesic coordinate system is in general valid in a sufficiently narrow worldtube along the timelike worldline of the observer.

the hypersurface orthogonal to the worldline is Euclidean space and one can describe some event on this hypersurface at $x^{\mu}$ to be at $X^{\mu}$, where $x^{\mu}$ and $X^{\mu}$ are connected via $X^{0}=c \tau$ and

$$
x^{\mu}=\bar{x}^{\mu}(\tau)+X^{i} \lambda^{\mu}{ }_{(i)}(\tau),
$$

where $\bar{x}^{\mu}$ represents the position of the accelerated observer.

From (7) we can derive (compare also with [2] and references therein) the relation

$$
\begin{aligned}
d x^{\mu} & =\frac{1}{c} \frac{d \bar{x}^{\mu}}{d \tau} d X^{0}+d X^{i} \lambda^{\mu}{ }_{(i)}+X^{i} d \lambda^{\mu}{ }_{(i)} \\
& =\lambda^{\mu}{ }_{(0)} d X^{0}+d X^{i} \lambda^{\mu}{ }_{(i)}+\frac{1}{c} X^{i} d X^{0}\left[\Phi_{i}{ }^{0} \lambda^{\mu}{ }_{(0)}+\Phi_{i}{ }^{j} \lambda^{\mu}{ }_{(j)}\right] \\
& =\left[\left(1+\frac{\vec{a} \cdot \vec{X}}{c^{2}}\right) \lambda^{\mu}{ }_{(0)}+\frac{1}{c}(\vec{\Omega} \times \vec{X})^{i} \lambda^{\mu}{ }_{(i)}\right] d X^{0}+\lambda^{\mu}{ }_{(i)} d X^{i},
\end{aligned}
$$

and hence the metric is

$$
\begin{aligned}
d s^{2} & =\eta_{\mu \nu} d x^{\mu} d x^{\nu} \\
& =-\left[\left(1+\frac{\vec{a} \cdot \vec{X}}{c^{2}}\right)^{2}-\frac{1}{c^{2}}(\vec{\Omega} \times \vec{X})^{2}\right]\left(d X^{0}\right)^{2}+\frac{2}{c}(\vec{\Omega} \times \vec{X}) \cdot d \vec{X} d X^{0}+\delta_{i j} d X^{i} d X^{j}
\end{aligned}
$$


Since we started from a global inertial frame in Minkowski spacetime, the spatial part of the line element yields Euclidean space with its origin occupied by the accelerated observer.

This set of coordinates is limited. If we follow the above procedure for two different times of the accelerated observer, our new coordinates may not be unique, see figure 1 . Since we cannot accept two sets of coordinates in the same system for one event, we have to require that the laboratory be sufficiently small. The charts for our coordinates cannot be global for accelerated observers. In fact, such geodesic coordinates are admissible as long as

$$
\left(1+\frac{\vec{a} \cdot \vec{X}}{c^{2}}\right)^{2}>\frac{1}{c^{2}}(\vec{\Omega} \times \vec{X})^{2} .
$$

Thus in the discussion of the admissibility of the geodesic coordinates, two independent acceleration lengths must be considered: the translational acceleration length $c^{2} / a$ and the rotational acceleration length $c / \Omega$ that appear in equation (10).

The acceleration radii are connected with the domain of applicability of the geodesic coordinate system around the reference accelerated observer. It turns out that these acceleration lengths have another independent and much more fundamental significance in terms of the local measurements of the accelerated observer following the reference trajectory [3, 过. This basic issue is discussed in section 3 .

It is important to remark here that one may use other (more complicated) accelerated coordinate systems; however, these have their attendant difficulties [5]. A discussion of these problems is beyond the scope of this paper; therefore, we limit our considerations here to geodesic coordinate systems.

Length scales for accelerated observers

The translational and rotational "accelerations" $a_{i}$ and $\Omega^{k}$ depend in general on both the velocity and the acceleration of the observer. We therefore construct the scalar invariants of the antisymmetric tensor $\Phi_{\alpha \beta}$, which are then independent of the (coordinate-dependent) velocity:

$$
\begin{gathered}
I=\frac{1}{2 c^{2}} \Phi_{\alpha \beta} \Phi^{\alpha \beta}=-\frac{a^{2}}{c^{4}}+\frac{\Omega^{2}}{c^{2}}, \\
I^{*}=\frac{1}{4 c^{2}} \Phi_{\alpha \beta}^{*} \Phi^{\alpha \beta}=-\frac{\vec{a}}{c^{2}} \cdot \frac{\vec{\Omega}}{c},
\end{gathered}
$$

where $\Phi_{\alpha \beta}^{*}$ is the dual of $\Phi_{\alpha \beta}$, i. e. $\Phi_{\alpha \beta}^{*}=\epsilon_{\alpha \beta \gamma \delta} \Phi^{\gamma \delta}$. We define the finite lengths $|I|^{-1 / 2}$ and $\left|I^{*}\right|^{-1 / 2}$ as the proper acceleration lengths.

Let us now see how long these lengths are in typical situations on the earth. For the translational acceleration length on the earth's surface we get $\left(a=9.8 \mathrm{~m} / \mathrm{s}^{2}, \Omega=0\right)$

$$
\frac{c^{2}}{a}=9.46 \cdot 10^{15} \mathrm{~m} \approx 1 \mathrm{ly},
$$

and for the rotational acceleration $\left(a=0, \Omega=\Omega_{\oplus}\right)$ the result is

$$
\frac{c}{\Omega}=4.1253 \cdot 10^{12} \mathrm{~m} \approx 27.5 \mathrm{AU} .
$$


Thus far we have discussed space-time measurements carried out by inertial observers. We must now consider the results of measurements carried out by an accelerated observer; moreover, it is important to see how such measurements are affected by the presence of an acceleration length $\mathcal{L}$.

\section{The Hypothesis of Locality}

In a spacetime diagram an inertial observer can be portrayed as a straight line. An observer that is linearly accelerated at some time will have a curved worldline. What will this accelerated observer measure? Typically, the Hypothesis of Locality [3, 佂 is tacitly assumed:

An accelerated observer measures the same physical results as a standard inertial observer that has the same position and velocity at the time of measurement.

The curved path of the observer is substituted by the straight line tangential to the curve at the time of measurement. The radius of curvature of the accelerated worldline is characterized by the acceleration length $\mathcal{L}$; the hypothesis of locality therefore assumes that locally $\mathcal{L}=\infty$. It is necessary to investigate if it is all right to reduce all measurements to the linear approximation, especially if we leave the infinitesimal neighborhood of an event and considering that realistic measuring devices are not infinitesimal.

The hypothesis of locality originates from Newtonian mechanics of classical point particles. The state of such a particle is given at each instant of time by its position and velocity. It follows that the hypothesis of locality is evidently valid in Newtonian mechanics and this explains the fact that no new physical assumption is needed in Newtonian physics to deal with accelerated systems.

It is important to recognize that the hypothesis of locality is crucial for the physical implementation of Einstein's heuristic principle of equivalence. This cornerstone of general relativity and the hypothesis of locality together imply that an observer in a gravitational field is pointwise inertial.

A restricted hypothesis of locality is the so-called clock hypothesis, which is a hypothesis of locality only concerned about the measurement of time. This hypothesis implies that a standard clock in fact measures $\tau, d \tau=\sqrt{1-\beta^{2}(t)} d t$, along its path; $\tau$ is then the proper time along this accelerated path. In the following sections, we set $\tau=0$ when $t=0$.

According to most experiments, the hypothesis of locality seems to be true. No experiment has yet shown the hypothesis of locality to be violated (outside of radiation effects). The main reason for this finding is that all relevant length scales in feasible experiments are very small in relation to the huge acceleration lengths of the tiny accelerations we usually experience. For instance, if we take the wavelength of light for a typical laboratory optics experiment, $\lambda \sim 10^{-7} \mathrm{~m}$, the factor $\lambda / \mathcal{L}$ is around $10^{-23}$ and $10^{-20}$ for translational and rotational accelerations, respectively. As long as all length scales are very small compared to the acceleration lengths, it seems reasonable 
to assume that differences between observations by accelerated and comoving inertial observers will also be very small.

It is the purpose of this paper to examine critically certain basic aspects of the hypothesis of locality in connection with the measurements of accelerated observers. To this end, we study in this work the measurement of length by accelerated observers. This choice is based in two considerations: (1) length measurement is a subject of crucial significance for a geometric theory of spacetime structure and (2) the hypothesis of locality must be applied not just at one event but at a continuous infinity of events for the determination of a finite length.

For practical purposes, the hypothesis of locality replaces the accelerated observer by an infinite sequence of otherwise identical momentarily comoving inertial observers. Every inertial observer is endowed with a natural orthonormal tetrad frame in Minkowski spacetime. Therefore, the same holds for an accelerated observer by the hypothesis of locality. It is then natural to interpret the results of section 2 as follows: The accelerated observer carries an orthonormal frame $\lambda^{\mu}{ }_{(\alpha)}(\tau)$ along its trajectory such that at each instant of its proper time $\tau$, the accelerated observer's temporal axis is $\lambda^{\mu}{ }_{(0)}$ and the spatial axes $\lambda^{\mu}{ }_{(i)}, i=1,2,3$, characterize the 3 -dimensional Euclidean space of this observer. Thus in the geodesic coordinate system adapted to this tetrad frame, the spatial part of the flat spacetime metric is always the 3-dimensional Euclidean space as in equation (9). Moreover, the local acceleration scales associated with the measurements of the observer are defined via equations (44)-(6) and (11). These have a physical significance that is distinct from the acceleration radii that mark the limits of the validity of the accelerated coordinate system as can be made clear by a simple example: For observers fixed on the rotating Earth, Earth-based coordinates are essentially valid only up to the light cylinder parallel to the Earth's axis and at a radius of $c / \Omega_{\oplus} \approx 28 \mathrm{AU}$ from it. This light cylinder, however, has no influence on the local measurements of the observer and the reception of astronomical data on the Earth. In contrast, the fact that such an observer is noninertial and therefore has local acceleration scales associated with it does affect its measurements as demonstrated by the phenomenon of spin-rotation coupling 6].

In our description of accelerated observers, an observer following a straight worldline in an inertial frame is not necessarily inertial. Consider, for example, an accelerated observer at rest in Minkowski spacetime that refers its observations to rotating axes. The observer's worldline is simply parallel to the time axis and the limitation of a geodesic coordinate system established around this observer does not arise from what is depicted in figure 1, but stems from the fact that observers at rest in the rotating frame would be moving relative to the reference observer at less than the speed of light only within its light cylinder. It follows that in the treatment of accelerated (i.e. noninertial) observers, the worldline as well as the spatial frame along the worldline must be taken into account. A more satisfactory frame bundle approach is indeed possible [2, [7], but such a treatment is beyond the scope of the present paper.

In the following sections, we consider specific thought experiments involving the measurement of distance between two accelerated observers. 


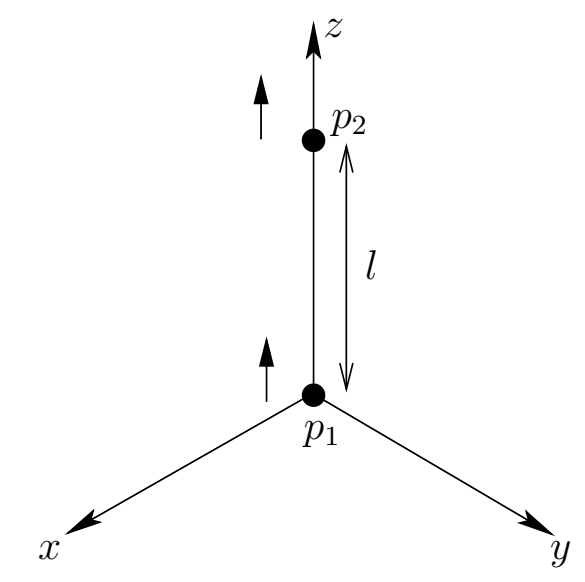

Fig. 2 Two observers a distance $l$ apart start accelerating from rest with identical acceleration profiles along the $z$-axis.

\section{Linear acceleration}

Consider two observers that are at rest in an inertial frame and a distance $l$ apart, see [3, 8]. At $t=0$ they both start to accelerate the same way, according to a preplanned acceleration profile. This type of thought experiment has been considered before [9]. We put one of the objects at the origin of our inertial coordinate system and the other one at $(0,0, l)$, and we assume that they accelerate linearly along the $z$-direction. For later calculations, we will specify the acceleration to be uniform along the $z$-axis, see figure 2. To avoid unphysical situations, we assume that the acceleration is always turned off at some finite time $t>0$.

An inertial observer at rest in the inertial frame describes the positions of the two accelerating objects to be

$$
z_{p_{1}}(t)=\int_{0}^{t} v(t) d t, \quad z_{p_{2}}(t)=l+\int_{0}^{t} v(t) d t .
$$

Hence, the distance between the accelerating objects stays constant, since $z_{p_{2}}(t)-$ $z_{p_{1}}(t)=l$.

Let us now investigate what comoving observers would measure for the distance between $p_{1}$ and $p_{2}$. The hypothesis of locality implies that both of the accelerated observers pass through the same infinite sequence of momentarily comoving inertial systems. The Lorentz transformation between the original inertial system and one of the comoving systems gives

$$
l^{\prime}=\frac{1}{\sqrt{1-\frac{v^{2}}{c^{2}}}} l=\gamma l,
$$


which we generalize to

$$
l^{\prime}=\frac{1}{\sqrt{1-\frac{v^{2}(t)}{c^{2}}}} l=\gamma(t) l .
$$

This has a simple physical interpretation: The Lorentz-Fitzgerald contracted distance between our accelerated objects is always $l$, hence the actual distance between them must be larger by the momentary Lorentz $\gamma$-factor. It is important to recognize that $p_{1}$ and $p_{2}$ could be any two points in a measuring device that is accelerated.

Specifically, let us imagine a set of accelerated observers populating the distance between $p_{1}$ and $p_{2}$ undergoing exactly the same motions as $p_{1}$ and $p_{2}$. At any given time $\hat{t}$, each of these observers is pointwise equivalent to a comoving inertial observer in accordance with the hypothesis of locality. The Lorentz transformation connecting the global background inertial frame with the rest frame of a comoving inertial observer at $(0,0, \hat{z})$ is given by

$$
\begin{aligned}
& c(t-\hat{t})=\hat{\gamma}\left(c t^{\prime}+\hat{\beta} z^{\prime}\right), \\
& x=x^{\prime}, \quad y=y^{\prime}, \quad z-\hat{z}=\hat{\gamma}\left(z^{\prime}+c \hat{\beta} t^{\prime}\right),
\end{aligned}
$$

where $\hat{\beta}$ and $\hat{\gamma}$ refer to the common speed of the system at $\hat{t}$. The consideration of length measurements of the standard observers in their inertial frames then leads to equation (16), i. e. the events $p_{1}:\left(c \hat{t}, 0,0, \hat{z_{1}}\right)$ and $p_{2}:\left(c \hat{t}, 0,0, \hat{z_{2}}\right)$ in the background global frame correspond to $p_{1}:\left(c t_{1}^{\prime}, 0,0, z_{1}^{\prime}\right)$ and $p_{2}:\left(c t_{2}^{\prime}, 0,0, z_{2}^{\prime}\right)$, where $c t_{1}^{\prime}=\hat{\gamma} \hat{\beta}(\hat{z}-$ $\left.\hat{z_{1}}\right), z_{1}^{\prime}=-\hat{\gamma}\left(\hat{z}-\hat{z_{1}}\right), c t_{2}^{\prime}=\hat{\gamma} \hat{\beta}\left(\hat{z}-\hat{z_{2}}\right)$, and $z_{2}^{\prime}=-\hat{\gamma}\left(\hat{z}-\hat{z_{2}}\right)$; therefore, $z_{2}^{\prime}-z_{1}^{\prime}=l^{\prime}=$ $\hat{\gamma}\left(\hat{z_{2}}-\hat{z_{1}}\right)=\hat{\gamma} l$.

For an alternative description, we should be able to replace the infinite sequence of inertial systems by one system in a continuously moving frame; for example, a coordinate system that has at its spatial origin one of the accelerating objects $\left(p_{1}\right)$. To this end, it is useful to introduce at this point the simplifying assumption that the observers are subject to uniform acceleration $g$. Observer $p_{1}$ thus follows a hyperbolic spacetime trajectory given by

$$
t=\frac{c}{g} \sinh \left(\frac{g \tau}{c}\right), \quad x=y=0, \quad z=z_{0}+\frac{c^{2}}{g}\left(-1+\cosh \left(\frac{g \tau}{c}\right)\right),
$$

where $z_{0}=0$ and $\tau$ is the proper time along the trajectory such that $\tau=0$ at $t=0$. The speed of the observer is thus $v=c \tanh \left(\frac{g \tau}{c}\right)$. We can construct an orthonormal tetrad frame along the reference trajectory such that at each instant it would coincide with the frame field of the momentary Lorentz transformation (17) and (18),

$$
\begin{aligned}
\lambda^{\mu}{ }_{(0)} & =(\gamma, 0,0, \gamma \beta), \\
\lambda^{\mu}{ }_{(1)} & =(0,1,0,0), \\
\lambda^{\mu}{ }_{(2)} & =(0,0,1,0), \\
\lambda^{\mu}{ }_{(3)} & =(\gamma \beta, 0,0, \gamma),
\end{aligned}
$$

where $\beta=\tanh \left(\frac{g \tau}{c}\right)$ and $\gamma=\cosh \left(\frac{g \tau}{c}\right)$. It follows from the hypothesis of locality that this is in fact the tetrad frame of the accelerated observer. Using this tetrad 
frame in equations (4)-(6) reveals that $\vec{a}=(0,0, g)$ and $\vec{\Omega}=\overrightarrow{0}$, so that the only proper acceleration length associated with the observer is $\mathcal{L}=\frac{c^{2}}{g}$, as expected. The spatial frame is in fact nonrotating, i. e. it is Fermi-Walker transported along the trajectory, so that the geodesic coordinate system constructed on this basis is a Fermi system.

According to equation (7), the relationship between the global inertial coordinates $x^{\mu}=(c t, x, y, z)$ and Fermi coordinates $X^{\mu}=(c T, X, Y, Z)$ along $p_{1}$ is given by

$$
c t=\left(Z+\frac{c^{2}}{g}\right) \sinh \left(\frac{g T}{c}\right), \quad x=X, \quad y=Y, \quad z=\left(Z+\frac{c^{2}}{g}\right) \cosh \left(\frac{g T}{c}\right)-\frac{c^{2}}{g}+z_{0},
$$

so that $p_{1}$ is always at the spatial origin of the Fermi system with $T=\tau$ and $z_{0}=0$. If the positions of the two accelerating objects in the original inertial frame at a time $\bar{t}$ are given by $p_{1}:(c \bar{t}, 0,0, \bar{z})$ and $p_{2}:(c \bar{t}, 0,0, l+\bar{z})$, then the corresponding positions in the moving coordinate system are $p_{1}:(c T, 0,0,0)$ and $p_{2}:\left(c T_{2}, 0,0, L\right)$. From equation (21) we get the relations

$$
c \bar{t}=\frac{c^{2}}{g} \sinh \left(\frac{g T}{c}\right), \quad \bar{z}=\frac{c^{2}}{g}\left[\cosh \left(\frac{g T}{c}\right)-1\right]
$$

and

$$
c \bar{t}=\left(L+\frac{c^{2}}{g}\right) \sinh \left(\frac{g T_{2}}{c}\right), \quad \bar{z}+l=\left(L+\frac{c^{2}}{g}\right) \cosh \left(\frac{g T_{2}}{c}\right)-\frac{c^{2}}{g} .
$$

Using $\cosh ^{2} \Theta-\sinh ^{2} \Theta=1$ in the last equation yields

$$
\left(L+\frac{c^{2}}{g}\right)^{2}=\left(l+\frac{c^{2}}{g}+\bar{z}\right)^{2}-c^{2} \bar{t}^{2}
$$

then, substituting for $\bar{t}$ and $\bar{z}+\frac{c^{2}}{g}$ using (22) leads to

$$
\left(L+\frac{c^{2}}{g}\right)^{2}=l^{2}+2 l \frac{c^{2}}{g} \cosh \left(\frac{g T}{c}\right)+\left(\frac{c^{2}}{g}\right)^{2}
$$

and this gives after some algebra

$$
L=\frac{c^{2}}{g}\left[\sqrt{1+2 \epsilon \gamma+\epsilon^{2}}-1\right]=\frac{l^{\prime}}{\gamma \epsilon}\left[\sqrt{1+2 \epsilon \gamma+\epsilon^{2}}-1\right]
$$

with $\epsilon=l / \frac{c^{2}}{g}$ and $\gamma=\cosh \left(\frac{g T}{c}\right)$. The parameter $\epsilon$ compares the length $l$ with the acceleration length in this case. For $\epsilon \gtrsim 1$, equation (26) implies that $L$ and $l^{\prime}$ can be very different; therefore, let us assume that $\epsilon \ll 1$. We now can compare $L$ with $l^{\prime}$, after applying the approximation $\sqrt{1+x}=1+\frac{1}{2} x-\frac{1}{8} x^{2}+\frac{1}{16} x^{3}+\mathcal{O}\left(x^{4}\right)$ for $|x|<1$,

$$
\frac{L}{l^{\prime}}=1-\frac{1}{2} \beta^{2} \gamma \epsilon+\frac{1}{2} \beta^{2} \gamma^{2} \epsilon^{2}+\mathcal{O}\left(\epsilon^{3}\right) .
$$


The length $L$ measured from $p_{1}$ in this accelerated frame differs from the length $l^{\prime}$ measured in a comoving inertial frame, if the length $l$ is not negligibly small in comparison to the acceleration length.

We now can change positions in this accelerated frame and investigate what length is measured from position $p_{2}$. Observer $p_{2}$ also follows a hyperbolic trajectory given by equation (19) with $z_{0}=l$. The corresponding transformation between inertial coordinates and Fermi coordinates is given by (21) with $z_{0}=l$. If the positions of the two accelerating objects in the original inertial frame at a time $\bar{t}$ are now given as before by $p_{1}:(c \bar{t}, 0,0, \bar{z})$ and $p_{2}:(c \bar{t}, 0,0, l+\bar{z})$, then the corresponding positions in the moving Fermi coordinate system are $p_{1}:\left(c T_{1}, 0,0,-L^{\prime}\right)$ and $p_{2}:(c T, 0,0,0)$. From equation (21) we get the relations

$$
c \bar{t}=\left(\frac{c^{2}}{g}-L^{\prime}\right) \sinh \left(\frac{g T_{1}}{c}\right), \quad \bar{z}-l=\left(\frac{c^{2}}{g}-L^{\prime}\right) \cosh \left(\frac{g T_{1}}{c}\right)-\frac{c^{2}}{g}
$$

and just as in equation (22),

$$
c \bar{t}=\frac{c^{2}}{g} \sinh \left(\frac{g T}{c}\right), \quad \bar{z}=\frac{c^{2}}{g} \cosh \left(\frac{g T}{c}\right)-\frac{c^{2}}{g} .
$$

Using $\cosh ^{2} \Theta-\sinh ^{2} \Theta=1$ in equation (28) yields

$$
\left(\frac{c^{2}}{g}-L^{\prime}\right)^{2}=\left(\frac{c^{2}}{g}+\bar{z}-l\right)^{2}-c^{2} \vec{t}^{2}
$$

which after substituting for $\bar{t}$ and $\bar{z}+\frac{c^{2}}{g}$ using (29) leads to

$$
\left(\frac{c^{2}}{g}-L^{\prime}\right)^{2}=l^{2}-2 l \frac{c^{2}}{g} \cosh \left(\frac{g T}{c}\right)+\left(\frac{c^{2}}{g}\right)^{2},
$$

and this gives after some algebra

$$
L^{\prime}=\frac{c^{2}}{g}\left[1-\sqrt{1-2 \epsilon \gamma+\epsilon^{2}}\right]=\frac{l^{\prime}}{\gamma \epsilon}\left[1-\sqrt{1-2 \epsilon \gamma+\epsilon^{2}}\right]
$$

with $\epsilon=l / \frac{c^{2}}{g}$ and $\gamma=\cosh \left(\frac{g T}{c}\right)$ as above. Again, for $\epsilon \ll 1$ let us now compare $L^{\prime}$ with $l^{\prime}$, after applying the approximation $\sqrt{1-x}=1-\frac{1}{2} x-\frac{1}{8} x^{2}-\frac{1}{16} x^{3}+\mathcal{O}\left(x^{4}\right)$ for $|x|<1$,

$$
\frac{L^{\prime}}{l^{\prime}}=1+\frac{1}{2} \beta^{2} \gamma \epsilon+\frac{1}{2} \beta^{2} \gamma^{2} \epsilon^{2}+\mathcal{O}\left(\epsilon^{3}\right) .
$$

The length $L^{\prime}$ measured from $p_{2}$ in this accelerated frame differs from the length $L$ (in fact, $L^{\prime}$ is larger than $L$ for $0<\epsilon<1$ ) and from the length $l^{\prime}$, if the length $l$ is not negligible compared to the acceleration length.

Let us now take another approach, based on our operational definition of length using electromagnetic signals: We want to measure the length by timing light rays. 
The relation between the measured time and the length can then be derived from the metric (9) for our case:

$$
d s^{2}=-\left(1+\frac{g X^{3}}{c^{2}}\right)^{2}\left(d X^{0}\right)^{2}+\delta_{i j} d X^{i} d X^{j} .
$$

For light rays along the $X^{3}$ - or $Z$-axis, $d s^{2}=0, d X^{1}=0$, and $d X^{2}=0$, and therefore:

$$
d Z= \pm\left(1+\frac{g Z}{c^{2}}\right) c d T .
$$

After integration we get

$$
c T+\text { constant }= \pm \frac{c^{2}}{g} \ln \left(1+\frac{g Z}{c^{2}}\right) .
$$

From the viewpoint of observer $p_{1}$, i.e. in the Fermi frame in which $p_{1}$ is at rest, let us suppose that the signal is emitted at time $T_{1}^{-}$from $Z=0$ such that the light travels the distance $Z: 0 \rightarrow L$ and arrives at time $T_{2}$ at $p_{2}$, since that is the position of $p_{2}:\left(T_{2}, 0,0, L\right)$ when the light arrives, i.e. $c \ln \left(1+g L / c^{2}\right)=g\left(T_{2}-T_{1}^{-}\right)$, and then back along $Z: L \rightarrow 0$, if we assume that the light is reflected by $p_{2}$ without delay so that it returns to $p_{1}$ at $T_{1}^{+}$such that $c \ln \left(1+g L / c^{2}\right)=g\left(T_{1}^{+}-T_{2}\right)$. Let us note that $T_{2}=\left(T_{1}^{+}+T_{1}^{-}\right) / 2$, which is the standard synchronization condition for distant events. With $L^{*}=c\left(T_{1}^{+}-T_{1}^{-}\right) / 2=c^{2} / g \ln \left(1+g L / c^{2}\right)$ for the length determined by light-ray timing, we find that $L^{*}<L$, where $L$ is determined by rulers in the accelerated system based on the hypothesis of locality; specifically, we get using (27)

$$
L^{*}=\frac{l^{\prime}}{\gamma \epsilon} \ln \left(1+\gamma \epsilon-\frac{1}{2} \gamma^{2} \beta^{2} \epsilon^{2}+\mathcal{O}\left(\epsilon^{3}\right)\right) .
$$

With $\ln (1+x)=x-\frac{1}{2} x^{2}+\mathcal{O}\left(x^{3}\right)$ for $-1<x \leq 1$, we finally find

$$
\frac{L^{*}}{l^{\prime}}=1-\frac{1}{2} \gamma \epsilon\left(1+\beta^{2}\right)+\mathcal{O}\left(\epsilon^{2}\right) \text {, }
$$

yet another result for the measured length if $\epsilon \neq 0$.

From the viewpoint of observer $p_{2}$, i.e. in the Fermi frame in which $p_{2}$ is at rest, the thought experiment can be repeated by sending the light signal from $p_{2}$ to $p_{1}$ and back without delay; in this case, a similar analysis holds except that we have to use $L^{\prime}$ instead of $L$ in the expression corresponding to $L^{*}$. The calculation for this case yields using (33)

$$
\frac{L^{\prime *}}{l^{\prime}}=1-\frac{1}{2} \frac{\epsilon}{\gamma}+\mathcal{O}\left(\epsilon^{2}\right) \text {. }
$$

It follows from these results that consistency can be achieved only if $\epsilon=g l / c^{2} \ll 1$ is below the level of sensitivity of the measurements of the accelerated observers.

It is possible to generalize our approach to arbitrary accelerated systems: Imagine two observers that are initially at rest in an inertial frame and subsequently move in 


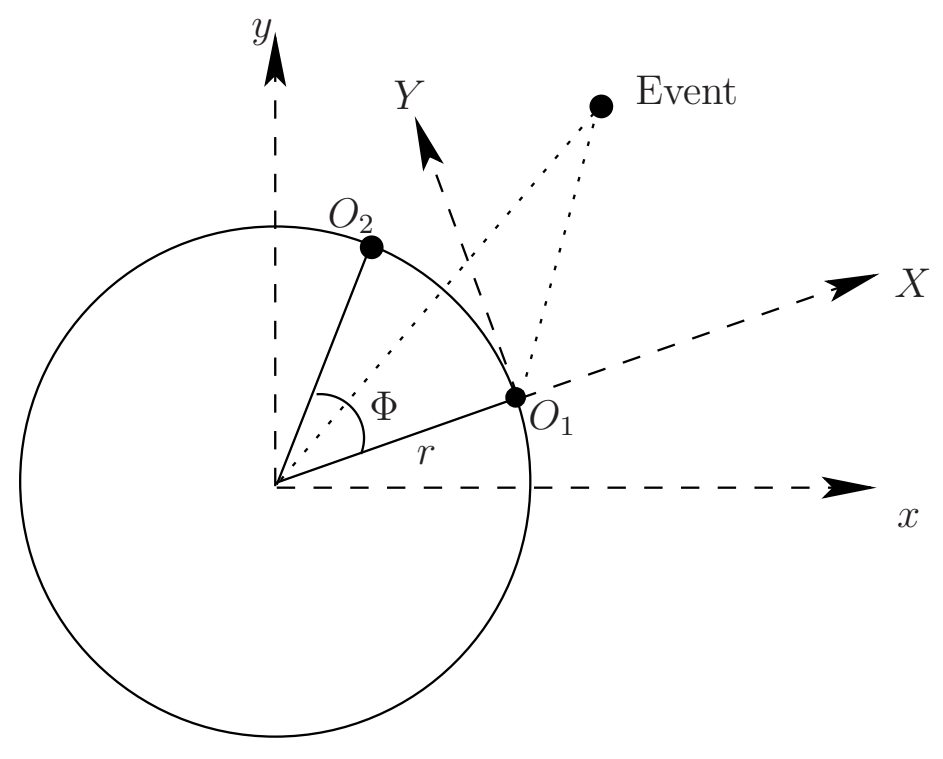

Fig. 3 Two observers uniformly rotating on a circle of radius $r$ with azimuthal angles $\phi_{1}=\Omega_{0} t$ and $\phi_{2}=\Omega_{0} t+\Phi$. An event can be described in the inertial frame $(c t, x, y, z)$ and in a rotating geodesic coordinate system $(c T, X, Y, Z)$.

exactly the same way for $t>0$. A vector analogue of equation (14) then implies that $\vec{x}_{p_{2}}(t)-\vec{x}_{p_{1}}(t)=\vec{x}_{p_{2}}(0)-\vec{x}_{p_{1}}(0)$, so that the Euclidean length between them remains the same as measured in the inertial frame. The determination of the distance between them as measured by the accelerated observers can be discussed as in the foregoing treatment. On the other hand, it is more interesting to consider a situation where the distance between the accelerated observers is defined along a curve rather than a straight line such as for two points fixed on the rotating Earth. Therefore, in the following section we consider rotating observers and assume that the rate of rotation is uniform for the sake of simplicity.

\section{Rotational acceleration}

We consider two observers $O_{1}$ and $O_{2}$ that rotate uniformly with angular velocity $\Omega_{0}$ on a circle with radius $r$ and with a constant angle $\Phi$ between them as in figure 3 . An inertial observer at rest in the global inertial frame would describe the arclength between the observers to have a constant length of $l=r \Phi$.

Let us now again investigate what comoving observers measure. For the sake of concreteness, we imagine a set of rotating observers populating the circle between $O_{1}$ and $O_{2}$ undergoing exactly the same motions as $O_{1}$ and $O_{2}$. The hypothesis of locality allows us to construct an infinite sequence of momentarily comoving inertial observers tangential to particles on the arc between the two circling observers. The Lorentz 
transformation between the original inertial observers at rest and one of the comoving inertial observers gives infinitesimally

$$
d l^{\prime}=\frac{1}{\sqrt{1-\frac{v^{2}}{c^{2}}}} d l=\gamma d l,
$$

with $v=r \Omega_{0}$. While $\gamma$ in the case of uniform linear acceleration was changing, it is constant here. By integrating over the comoving inertial observers we get $l^{\prime}=\gamma l$ for the arclength between the objects. The physical interpretation is the same as in the case of linear acceleration: The Lorentz-Fitzgerald contracted arclength between our rotating objects is always $l$, hence the actual arclength between them must be larger by the Lorentz $\gamma$-factor. Again, it is important to recognize that $O_{1}$ and $O_{2}$ could be any two points in a rotating measuring device.

As in the case of linear acceleration, we now attempt an alternative description that is also based on the hypothesis of locality and replace the infinite sequence of momentarily comoving inertial frames by one continuously moving frame, for example, the geodesic coordinate system around the worldline of one of the rotating observers.

Consider a rotating observer in the $(c t, x, y, z)$ coordinate system as in figure 3 . It turns out that the natural orthonormal tetrad frame of such an observer is given by [4]

$$
\begin{aligned}
& \lambda^{\mu}{ }_{(0)}=\gamma(1,-\beta \sin \varphi, \beta \cos \varphi, 0), \\
& \lambda^{\mu}{ }_{(1)}=(0, \cos \varphi, \sin \varphi, 0), \\
& \lambda^{\mu}{ }_{(2)}=\gamma(\beta,-\sin \varphi, \cos \varphi, 0), \\
& \lambda^{\mu}{ }_{(3)}=(0,0,0,1) .
\end{aligned}
$$

where $\varphi$ is the azimuthal angle of the observer such that $\frac{d \varphi}{d t}=\Omega_{0}, \beta=r \Omega_{0} / c$ and $\gamma$ is the corresponding Lorentz factor. In this case, the components of the acceleration tensor (6) turn out to be $\vec{a} / c=\left(-\beta \gamma^{2} \Omega_{0}, 0,0\right)$ corresponding to the centripetal acceleration and the rotation $\vec{\Omega}=\left(0,0, \gamma^{2} \Omega_{0}\right)$ of the spatial frame with frequency $\gamma^{2} \Omega_{0}$ about the nonrotating triad that represents ideal gyroscope directions [4]. To determine the proper acceleration length in this case, we note that $I=\frac{\gamma^{2} \Omega_{0}^{2}}{c^{2}}$ and $I^{*}=0$. Thus $\mathcal{L}=\frac{c}{\gamma \Omega_{0}}$, where $\gamma \Omega_{0}=\frac{d \varphi}{d \tau}$ is the proper rotation frequency of the observer.

Let us now construct a geodesic coordinate system based on the tetrad frame (41) for observer $O_{1}$, i.e. we set $\varphi=\varphi_{1}=\Omega_{0} t$ in (41). In equation (7), the worldline $\bar{x}^{\mu}(\tau)$ of $O_{1}$ is therefore given in $(c t, x, y, z)$ coordinates by $O_{1}:\left(c t, r \cos \varphi_{1}, r \sin \varphi_{1}, 0\right)$, where $t=\gamma \tau$ and $\varphi_{1}=\gamma \Omega_{0} \tau$. Hence equation (1) implies that the rotating geodesic coordinate system $(c T, X, Y, Z)$ is related to the original inertial coordinates $(c t, x, y, z)$ by (compare figure 3)

$$
\begin{aligned}
& c t=\gamma(c T+\beta Y), \quad x=(X+r) \cos \left(\gamma \Omega_{0} T\right)-\gamma Y \sin \left(\gamma \Omega_{0} T\right), \\
& y=\gamma Y \cos \left(\gamma \Omega_{0} T\right)+(X+r) \sin \left(\gamma \Omega_{0} T\right), \quad Z=z .
\end{aligned}
$$

Consider now an observer $O:(c t, r \cos \varphi, r \sin \varphi, 0)$ on the arc between $O_{1}$ and $O_{2}$ at a given time $t$ with $\varphi=\Omega_{0} t+\phi$ such that the fixed angle $\phi$ could range from $\phi=0$ 
at $O_{1}$ to $\phi=\Phi$ at $O_{2}$. It follows from the coordinate transformation (42) that in the geodesic coordinate system $O:(c T, X, Y, 0)$, where

$$
X+r=r \cos \chi, \quad Y=\gamma^{-1} r \sin \chi
$$

Here $\chi$ is an angle defined by $\chi=\varphi-\gamma \Omega_{0} T$; therefore, using $\varphi=\Omega_{0} t+\phi$ and $t=\gamma T+\gamma \beta Y / c$ we find

$$
\chi-\beta^{2} \sin \chi=\phi \text {. }
$$

It follows that in the geodesic coordinate system, $O$ lies on an ellipse

$$
\frac{(X+r)^{2}}{r^{2}}+\frac{Y^{2}}{r^{2}\left(1-\beta^{2}\right)}=1
$$

with semimajor axis $r$, semiminor axis $\gamma^{-1} r$ and eccentricity $\beta=r \Omega_{0} / c$ as depicted in figure 4 . This figure should be compared and contrasted with figure 3 . The ellipse can be thought of as the circle of radius $r$ that is Lorentz-Fitzgerald contracted along the direction of motion (i.e. the $Y$-axis). The angle $\chi$ is similar to the eccentric anomaly in Keplerian motion and ranges from $\chi=0$ at $O_{1}$ to $\chi=\Delta$ at $O_{2}$, i.e.

$$
\Delta-\beta^{2} \sin \Delta=\Phi
$$

by equation (44). It is interesting to note that equation (44) is similar to the Kepler equation for elliptical motion in Newtonian gravity, except that in the Kepler equation the eccentricity $\beta$ takes the place of $\beta^{2}$ in (44). Moreover, for a given angle $\phi$, there is a unique angle $\chi$ for $0 \leq \beta^{2}<1$.

In the rotating geodesic coordinate system established around $O_{1}$, the distance from $O_{1}$ to $O_{2}$ along the elliptical arc is $D$,

$$
D=r \int_{0}^{\Delta} \sqrt{1-\beta^{2} \cos ^{2} \chi} d \chi
$$

which is in general different from $l^{\prime}=\gamma r \Phi$. For instance, for a fixed $\Phi, l^{\prime} \rightarrow \infty$ as $\beta \rightarrow 1$, while $D \rightarrow r(1-\cos \Delta)$ in this limit so that $D / l^{\prime} \rightarrow 0$. Moreover, $D$ is a monotonically increasing function of $\Phi$ for fixed $\beta$.

On the other hand, let us fix $\Phi$ at $\pi$ and note that when $\Phi=\pi, \Delta=\pi$ as well from equation (46); then, the half circumference of the ellipse is given by

$$
D=\pi r\left[1-\left(\frac{1}{2}\right)^{2} \beta^{2}-\left(\frac{1 \cdot 3}{2 \cdot 4}\right)^{2} \frac{\beta^{4}}{3}-\left(\frac{1 \cdot 3 \cdot 5}{2 \cdot 4 \cdot 6}\right)^{2} \frac{\beta^{6}}{5}-\mathcal{O}\left(\beta^{8}\right)\right]
$$

so that as $\beta$ goes from $0 \rightarrow 1$, the corresponding $D$ decreases from $\pi r \rightarrow 2 r$ and $D / l^{\prime}$ goes from $1 \rightarrow 0$. To understand this variation intuitively, we note that $\beta \gamma=r / \mathcal{L}$. That is,

$$
\frac{l^{\prime}}{\mathcal{L}}=\beta \Phi
$$




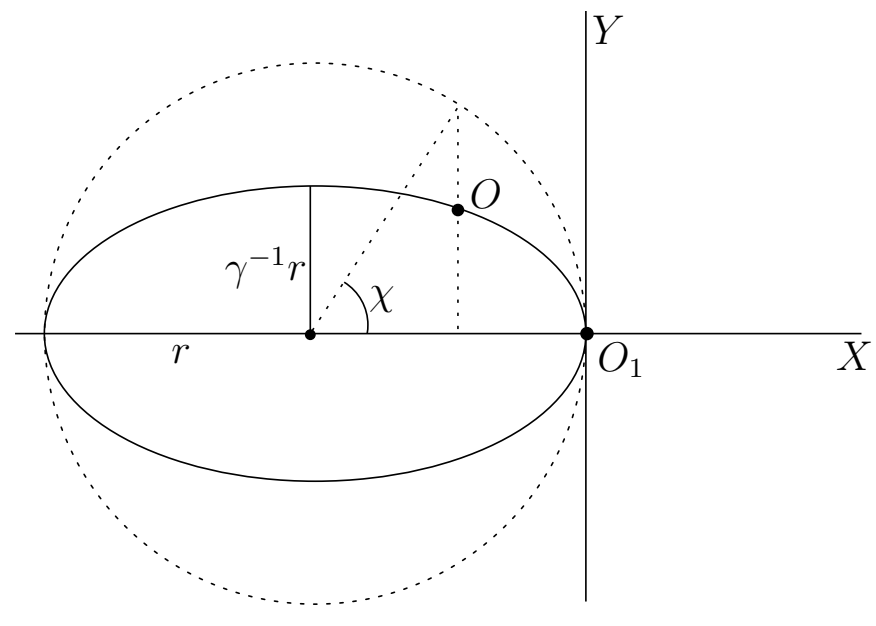

Fig. 4 The observers $O_{1}$ and $O$ are depicted here from the standpoint of the geodesic coordinate system established around the worldline of $O_{1}$. The ellipse is given by equation (45) and $O$ would range from $O_{1}$ at $\chi=0$ up to $O_{2}$ at $\chi=\Delta$, where $\Delta-\beta^{2} \sin \Delta=\Phi$. The length of the elliptical arc from $O_{1}$ to $O_{2}$ is given by $D$ in equation (47). This is naturally related to elliptic integrals; that is, $D=r\left[E\left(\frac{\pi}{2}, \beta\right)-E\left(\frac{\pi}{2}-\Delta, \beta\right)\right]$, where $E(\varphi, k)=$ $\int_{0}^{\varphi} \sqrt{1-k^{2} \sin ^{2} \alpha} d \alpha$ is the elliptic integral of the second kind.

in the case under consideration here with $0 \leq \Phi<2 \pi$. Thus, when the circular orbit is much smaller than the acceleration length of the observer, $\beta \gamma=r / \mathcal{L} \ll 1$, expanding equation (47) in powers of $\beta^{2} \ll 1$ we find that

$$
\frac{D}{l^{\prime}}=1-\frac{3}{4} \beta^{2}\left(1+\frac{\sin 2 \Phi-8 \sin \Phi}{6 \Phi}\right)+\mathcal{O}\left(\beta^{4}\right)
$$

where $\Phi=\frac{l}{r}$. When the radius of the circular orbit is much smaller than the acceleration length of the observer, $D \approx l^{\prime}$; however, the deviation of $\frac{D}{l^{\prime}}$ from unity cannot be neglected for $\beta \rightarrow 1$.

If the geodesic coordinate system is established along the worldline of the observer $\mathrm{O}_{2}$ instead, then the arclength from $\mathrm{O}_{2}$ to $O_{1}$ in the accelerated system turns out to be $D$ as well due to the symmetry of the uniformly rotating configuration depicted in figure 3 .

Considering our results, it is necessary to recognize that there is no unique answer for event distances when the observer is accelerated. We do not have a theory that gives us the precise distance on the Earth between Cologne (Germany) and Columbia (Missouri), for example, since the Earth rotates. Of course, $\epsilon=\beta \gamma \Phi$ is typically very small, since it compares $l$ with the very large acceleration length $\mathcal{L}$. For instance, for antipodal points along the equator, equation (50) implies that the difference between $D$ and $l^{\prime}$ amounts to only a distance of the order of $10^{-3} \mathrm{~cm}$. 


\section{Discussion}

The main purpose of this work has been to demonstrate that within the confines of classical, i.e. nonquantum, physics there exist basic limitations on length measurement by accelerated observers in Minkowski spacetime that follow from the hypothesis of locality. Indeed, realistic accelerated coordinate systems suffer from limitations that are far more severe than those imposed by the requirement of the admissibility of such coordinates. That is, all distances in accelerated systems must in fact be negligibly small compared to the characteristic acceleration lengths of the observer.

Discussions of the quantum limitations of spacetime measurements are contained in [3] and [10]. Difficulties with the measurement of spatial distance in the general theory of relativity are treated in [11].

\section{References}

[1] R. E. Scherr, P. S. Shaffer, and S. Vokos: Student understanding of time in special relativity: Simultaneity and reference frames. Physics Education Research, American Journal of Physics, Supplement 69, S24-S35 (July 2001).

[2] F. W. Hehl, J. Lemke, and E. W. Mielke: Two lectures on fermions and gravity; In: Geometry and Theoretical Physics, Proceedings of the Bad Honnef School, 12-16 February 1990. J. Debrus and A.C. Hirshfeld, eds. (Springer, Heidelberg 1991) pp. 56-140.

[3] B. Mashhoon: Limitations of spacetime measurements; Phys. Lett. A143 (1990) 176182.

[4] B. Mashhoon: The Hypothesis of Locality in relativistic physics; Phys. Lett. A145 (1990) $147-153$.

[5] K.-P. Marzlin: What is the reference frame of an accelerated observer?; Phys. Lett. A215 (1996) 1-6.

[6] B. Mashhoon: On the coupling of intrinsic spin with the rotation of the Earth; Phys. Lett. A198 (1995) 9-13.

[7] U. Muench, F. W. Hehl, and B. Mashhoon: Acceleration-induced nonlocal electrodynamics in Minkowski spacetime; Phys. Lett. A271 (2000) 8-15.

[8] B. Mashhoon: Measurement theory and general relativity; In: Black Holes: Theory and Observation: Proceedings of the $179^{\text {th }}$ W.E. Heraeus Seminar, held August 1997 in Bad Honnef, Germany. F. W. Hehl et al.(eds). (Springer, Heidelberg 1998) pp. 269-284.

[9] J.S. Bell: Speakable and unspeakable in quantum mechanics (Cambridge University Press, Cambridge, 1987).

[10] H. Salecker and E.P. Wigner: Quantum limitations of the measurement of space-time distances; Phys. Rev. 109 (1958) 571-577.

[11] H.-J. Schmidt: How should we measure spatial distances?; Gen. Rel. Grav. 28 (1996) 899-903. 